Musées, Patrimoine et Culture scientifiques et techniques

$140 \mid 2012$

mars-avril 2012

\title{
Conservation préventive et développement durable
}

Preventive conservation and sustainable development

\section{Frédérique Vincent}

\section{OpenEdition \\ Journals}

Édition électronique

URL : http://journals.openedition.org/ocim/1055

DOI : $10.4000 /$ ocim. 1055

ISSN : 2108-646X

\section{Éditeur}

OCIM

Édition imprimée

Pagination : 27-31

ISSN : 0994-1908

Référence électronique

Frédérique Vincent, "Conservation préventive et développement durable », La Lettre de I'OCIM [En ligne], 140 | 2012, mis en ligne le 01 mars 2014, consulté le 01 mai 2019. URL : http:// journals.openedition.org/ocim/1055; DOI : 10.4000/ocim.1055 


\section{Conservation préventive et développement durable}

\section{Frédérique Vincent *}

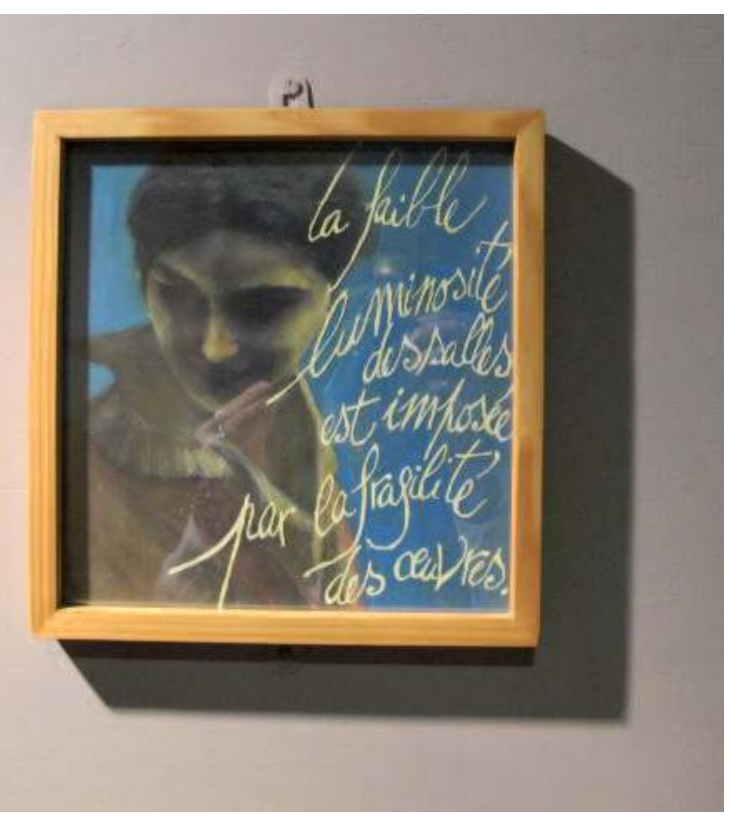

Sensibilisation du public à la fragilité du patrimoine, dans les salles d'exposition du musée de la Vie Bourguignonne à Dijon ๑ Frédérique Vincent

* Frédérique Vincent est consultante en conservation préventive et restauratrice d'objets ethnographiques, f.vincent@numericable.com
Comment réunir les objectifs de la conservation préventive et ceux du développement durable ? À partir de trois thèmes - l'architecture, le climat et les pratiques professionnelles - cette contribution fait le point sur les bons comportements que les institutions muséales doivent s'efforcer d'adopter dans ce domaine.

\author{
"Nous n'héritons pas de la Terre de nos ancêtres, \\ nous l'empruntons à nos enfants » \\ Antoine de Saint-Exupéry
}

De nos jours et dans le contexte environnemental et sociétal qui est le nôtre, la conservation du patrimoine ne peut plus faire abstraction de la question du développement durable. La définition conventionnelle retenue du développement durable a été formulée par la commission mondiale sur l'environnement et le développement des Nations Unies en 1987 : «C'est un développement qui répond aux besoins des générations $d u$ présent sans compromettre la capacité des générations futures à répondre aux leurs » (1). Cette définition a des résonances importantes avec les principes de la conservation du patrimoine, tels que l'ICOM les a définis, entre autres : "La politique du musée doit faire que les collections (permanentes et temporaires) et leurs informations associées, correctement consignées, soient transmises aux générations futures dans les meilleures conditions possibles, compte tenu des connaissances et des ressources disponibles » ${ }^{(2)}$.

Certains mots-clés associés au développement durable, sont utilisés en conservation du patrimoine : longterme, pérennité, prévention des risques, préservation, intégrité culturelle... 
Face au changement climatique, à la multiplication des catastrophes naturelles et industrielles, à la raréfaction des ressources naturelles..., mais aussi grâce à une meilleure connaissance des matériaux, à la prise en compte de l'évaluation des risques, aux progrès de la science et des techniques..., la réflexion en conservation s'oriente de plus en plus vers des solutions en adéquation avec le développement durable et le respect de l'environnement. Nous le verrons dans cet article à travers trois thèmes : l'architecture, le climat et les pratiques professionnelles.

Depuis quelques années, toute démarche de conservation est basée sur l'étude des collections (constats d'état, bilans sanitaires et évaluation des conditions de conservation), et sur la définition par des spécialistes des besoins de ces collections (choix des environnements climatiques, définition des interventions curatives ou de restauration nécessaires, principes de rangement en réserves ou de présentation en exposition, mise en place de systèmes de prévention, organisation de chantiers des collections...). Cette démarche de conservation s'appuie aussi sur l'analyse des risques que courent ces collections afin de définir des priorités, d'intervenir à bon escient et d'adapter au mieux l'environnement des collections au sens large et les habitudes de travail, en fonction des urgences établies.

Mais pour être adaptée et utile, cette démarche doit absolument prendre en compte les objectifs à court, moyen et long terme de l'institution patrimoniale publique ou privée, les réalités locales, les ressources disponibles, qu'elles soient humaines, financières, ou techniques et toute information ou élément permettant d'être en adéquation avec la situation.

\section{Conservation et architecture}

Depuis plusieurs années, on assiste en France à l'intégration de spécialistes en conservation préventive dans les équipes de programmation architecturale que ce soit pour la création ou la rénovation d'un bâtiment de musée, d'archives, de réserves... Cette compétence supplémentaire dans les équipes a permis de mieux comprendre, de définir et finalement de prendre en compte non seulement les besoins des collections (en termes de gestion, conditions climatiques, réserves, traitements...), mais aussi les besoins des professionnels travaillant sur ces collections. Des équipements (comme un quai de déchargement adapté aux collections), des locaux spécifiques (comme une quarantaine, un espace d'emballage) agencés pour faciliter le travail, des espaces simples d'entretien et de maintenance... contribuent à améliorer les conditions de travail des personnels et participent de cette manière au développement durable.

Grâce à cela, les projets architecturaux donnent naissance à des bâtiments bien pensés dès la conception, évitant ainsi au maximum les dysfonctionnements, les problèmes de conservation, les dépenses de fonctionnement démesurées ainsi que les coûts ultérieurs liés à des travaux d'adaptation des lieux en vue d'améliorer leur utilisation en tant qu'institution patrimoniale.

Afin de s'inscrire dans une démarche de développement durable, la conception architecturale doit aussi prendre en compte les spécificités du lieu de construction : construction sur pilotis en béton pour éviter les attaques de termites souterrains (exemple de réserve en Côte d'Ivoire), toitures débordantes pour éviter l'ensoleillement ou l'humidification des murs en fonction des périodes (Palais royaux d'Abomey, Bénin), ventilation naturelle pour éviter le confinement et les zones de concentration d'humidité (dépôt archéologique de Guyane), mise en place d'un système de résistance à l'arrachage des toits par les cyclones (musée de Tahiti et des Îles)...

En parallèle, la démarche de Haute Qualité Environnementale ou HQE ${ }^{(3)}$ se systématise. Les bâtiments deviennent économes en consommation d'énergie (basse consommation ou "zéro énergie ») ou même à énergie positive c'est-à-dire quilils produisent plus d'énergie qu'ils n'en consomment pour leur fonctionnement.

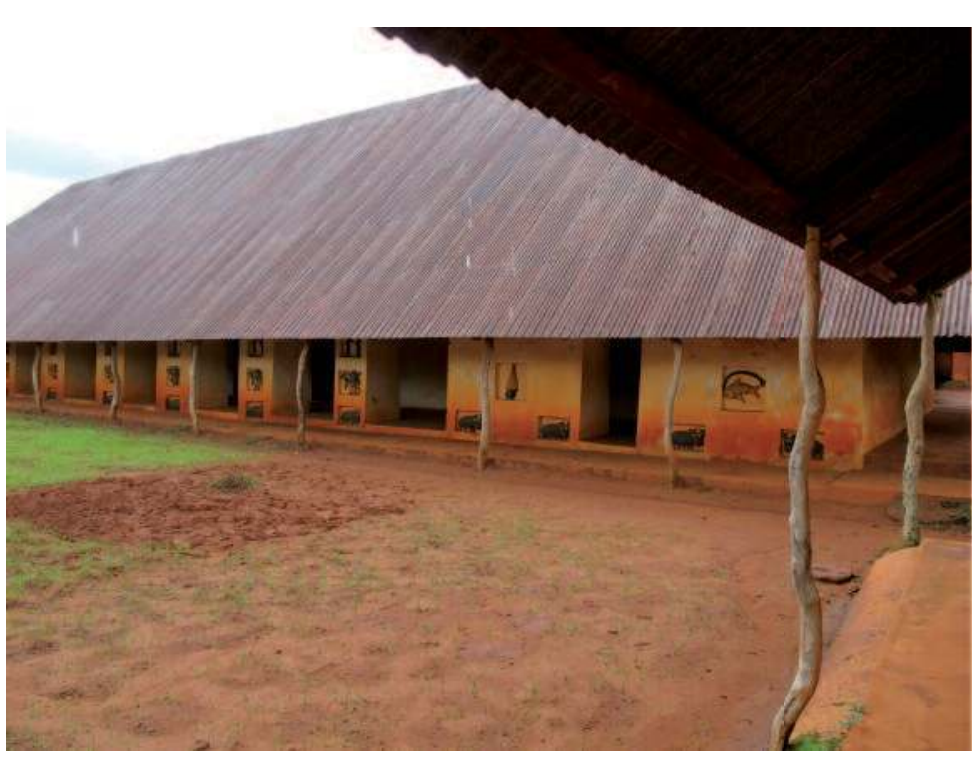

Toits débordants des Palais royaux d'Abomey au Bénin, sous la pluie. () Frédérique Vincent 
Dans cette perspective, l'architecte agit sur l'économie des énergies (eau, électricité, gaz...), l'amélioration de l'inertie du bâtiment et de la stabilité climatique par l'isolation et l'étanchéification, le choix de matériaux de construction et leur recyclage ${ }^{(4)}$, l'emploi de procédés de construction adaptés, l'utilisation de matériaux de finition (peintures, revêtements...) durables et non polluants, la mise en place d'équipements de détection systématiques (feu, intrusion-vol, inondations, insectes...), le choix de systèmes scénographiques adaptés à la bonne conservation, peu calorifiques et énergivores (éclairage avec des diodes électroluminescentes par exemple), une répartition des collections dans les différents espaces adaptée à leur sensibilité (les plus fragiles dans les espaces les plus stables)...

L'exemple du Centre de Conservation et d'Étude de Lons-le-Saunier, qui a fait l'objet d'un article dans la Lettre de l'OCIM répond à différents critères énoncés ci-dessus ${ }^{(5)}$. Ainsi, la mutualisation d'un lieu et de ressources humaines, financières et techniques pour des institutions différentes, la recherche de stabilité thermique pour assurer la conservation des collections tout en limitant la consommation d'énergie, la démarche d'empreinte écologique (6) basse passant par le choix des matériaux et la formation des ouvriers, et un bâtiment modulable en prévision du futur sont autant d'éléments participant au développement durable.

\section{Adaptation des normes climatiques}

Cette évolution des mentalités a aussi permis de mener une réflexion internationale sur les normes climatiques. Celles-ci étaient autrefois très rigides, et ce quel que soit le pays dans lequel étaient conservées les collections, par exemple $18^{\circ} \mathrm{C}$ et $50 \%$ d'humidité relative en réserves, quels que soient l'environnement climatique du lieu et la saison. L'assouplissement de ces normes climatiques ne s'est pas fait au détriment de la bonne conservation des collections. Au contraire, il s'est basé sur l'analyse de l'histoire matérielle des œuvres (historique de leurs conditions de conservation au cours du temps), sur l'étude de leur état de conservation et s'est appuyé sur les progrès de la recherche, menée par des institutions spécialisées comme l'Institut Canadien de Conservation ou le Centre de Recherches sur la Conservation des Collections (CRCC-CNRS) de Paris, apportant une meilleure connaissance de la sensibilité des matériaux constitutifs des œuvres.

Ces normes sont donc aujourd'hui plus souples, mieux adaptées aux spécificités et aux besoins des collections

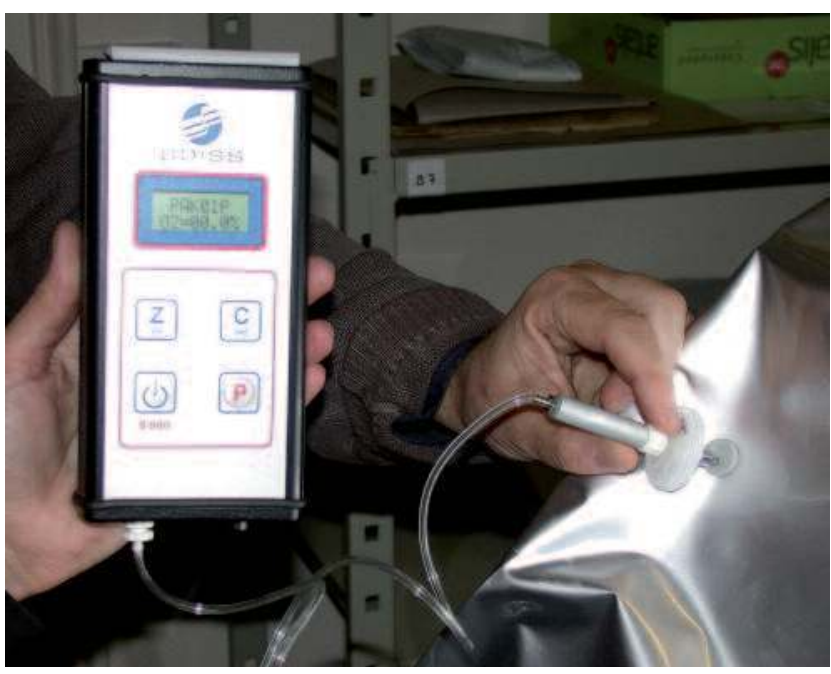

Mesure du taux d'oxygène $d^{\prime}$ une bulle de traitement de désinfestation par anoxie statique (c) Frédérique Vincent

et à leurs lieux de conservation et sont plus en adéquation avec l'environnement local, tout en prenant en compte les moyens techniques et les ressources financières locales ${ }^{(7)}$. Ainsi, on accepte facilement aujourd'hui qu'un lot de mobiliers archéologiques lapidaires ou céramiques ne soit pas conservé dans un environnement climatisé lorsqu'il est conservé dans un pays à climat tempéré, mais que les éléments en métal ou en bois soient dissociés et installés, eux, dans des espaces où l'humidité relative et la température sont traitées ou dans des microclimats (boîtes avec gel de silice par exemple).

On accepte aussi des réglages saisonniers, hiver et été, pour réduire la consommation énergétique. Ainsi, depuis plusieurs années, on reconnaît l'utilité de baisser les températures au Canada en hiver pour éviter la dégradation par l'humidité des édifices historiques. Cette baisse contribue en même temps à économiser l'énergie et à réduire les coûts.

Cette réflexion sur les normes climatiques permet aussi de ne pas choisir systématiquement un équipement de climatisation coûteux, énergivore et difficile à entretenir, qu'on a tendance à arrêter la nuit dans certains pays pour des raisons économiques, mais favorise le choix d'un traitement de l'air plus «simple» (rafraichissement et chauffage pour le bien-être humain par exemple). Des institutions, comme le Getty Conservation Institute (GCI) de Los Angeles, ont entrepris des recherches sur une gestion «mécanique » du climat, en utilisant des équipements "portables " comme des ventilateurs et des déshumidificateurs. Ainsi, le GCI a mis en place un système de contrôle du climat dans les institutions 


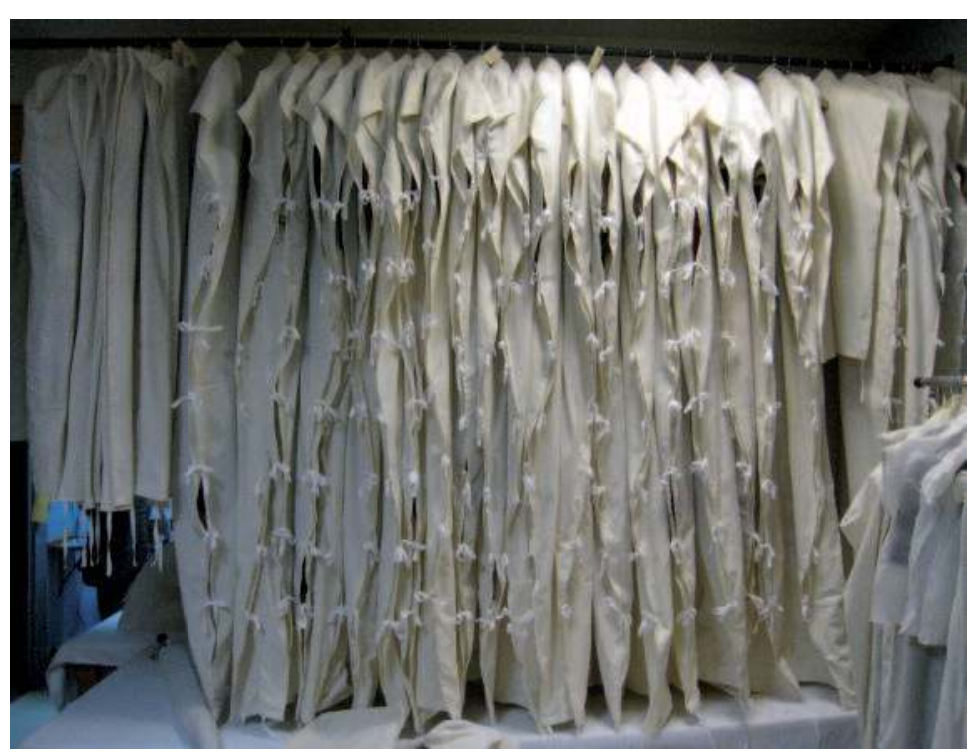

Housses en coton, réalisées en interne, au musée de la Vie bourguignonne à Dijon. (c) Patricia dal-Prà

patrimoniales de pays à climat tropical chaud et humide (Brésil, Tenerife...), sans utiliser d'air conditionné (voir exemple du musée Goeldi au Brésil) (8). Ces équipements, moins énergivores et plus économiques présentent l'avantage d'être plus faciles à entretenir que des centrales climatiques.

Bien entendu, tous ces aménagements ont pour objectif premier la bonne conservation des collections tout en concourant aux objectifs de meilleur respect de l'environnement et d'économie.

\section{Pratiques professionnelles}

Les professionnels d'institutions patrimoniales sont les principaux acteurs de la conservation du patrimoine et de la mise en place des principes du développement durable. À ce sujet, des groupes de travail ont été mis en place dans différents musées en France et dans le Monde. Les choix faits dans le contexte actuel doivent absolument être basés sur l'analyse des besoins des collections, mais aussi sur la rationalisation des ressources quelles qu'elles soient.

Ainsi, dans les pays où ils sont disponibles, l'utilisation de matériaux de conditionnement ou d'emballage à base de pétrole comme les plastiques (polyéthylène, polyester...), présente des avantages, malgré leur empreinte carbone. Dans le cadre d'une vision de la conservation à long terme, l'utilisation de matériaux pérennes, neutres, et adaptés à la conservation des collections est intéressante, plutôt que d'utiliser des matériaux de moins bonne qualité qu'il faudra remplacer à court ou moyen terme. Cependant, ce n'est pas parce que ces matériaux plastiques sont encore disponibles et relativement abordables qu'ils doivent être systématiquement utilisés. Leur emploi se doit d'être raisonné, à bon escient et des questions préalables doivent être posées pour choisir le type adapté : nature de l'utilisation (on pourra préférer un matériau d'origine naturelle comme le coton pour une protection contre la poussière), temps d'utilisation (matériau plus fin ou de moins bonne qualité si emploi à court terme), fragilité et nature des œuvres, mouvement des collections emballées ou simple stockage (dans ce cas des mousses moins épaisses peuvent être utilisées ou de simples calages), risques de chutes, de vibrations, d'empoussièrement... La question de leur réemploi, la volonté de limiter les déchets, favoriser le recyclage, encourager la mutualisation et la réutilisation... doivent aussi être envisagées en amont. À cet effet, il peut être décidé de ne pas utiliser d'adhésifs pour ne pas déchirer les matériaux comme le Tyvek ${ }^{(}$par exemple et pouvoir le réutiliser, ou de donner certains conditionnements qu'on n'utilise plus à d'autres institutions financièrement moins aisées.

Il est aussi important de s'adapter au contexte local. $\mathrm{Si}$ ces matériaux plastiques stables et neutres ne sont pas disponibles sur place, il n’est pas forcément nécessaire de les faire venir par avion (émission importante de gaz à effets de serre et coûts importants), mais on peut essayer, en étudiant les pratiques locales, de trouver des ressources et savoir-faire disponibles sur place.

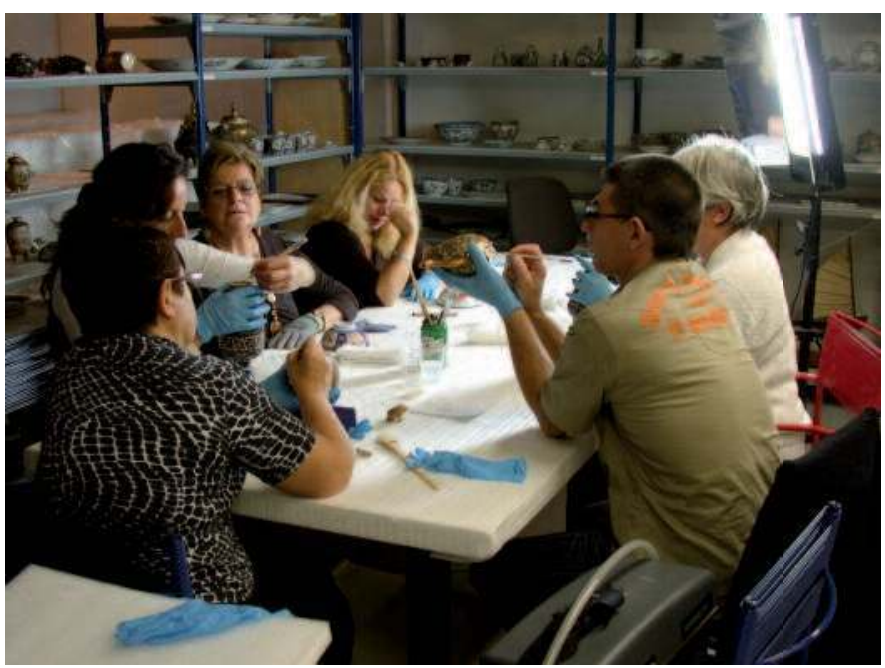

Formation du personnel et de bénévoles aux gestes de la conservation au musée des Beaux-Arts de Dunkerque (๑) Frédérique Vincent 
Dans le secteur des traitements curatifs et de la restauration, on cherche aujourd'hui à limiter l'emploi de solvants et produits chimiques, tels que des insecticides, pour leur préférer des produits naturels et des techniques non polluantes. Ainsi, l'oxyde d'éthylène, longtemps utilisé pour les désinfestations, a été remplacé petit à petit par l'anoxie statique ou dynamique (en utilisant des gaz inertes) et la congélation. Des recherches importantes sont aujourd'hui menées sur des traitements par la chaleur aux Pays-Bas ou au Canada. La prévention, lorsqu'elle est mise en place, est essentielle puisqu'elle permet de limiter les infestations et donc leur traitement.

En restauration, on préfère aujourd'hui, autant que possible, utiliser des traitements aqueux à base d'enzymes pour nettoyer les œuvres peintes plutôt que des solvants à base de pétrole, nocifs pour la santé et l'environnement.

La formation du personnel travaillant dans les institutions patrimoniales est aussi un vecteur important de la conservation et du développement durable. Ainsi, par exemple, si on sensibilise l'ensemble du personnel à la fragilité du patrimoine et si on lui explique les mécanismes de dégradation par la lumière, il trouvera toujours contraignant mais nécessaire de devoir fermer les volets ou les éclairages lorsque le public n'est pas là, que ce soit pour la conservation des collections ou pour les économies d'énergie.

Expliquer la nocivité de l'utilisation intempestive de certains produits d'entretien (comme les nettoyants pour le métal, les cires assouplissantes pour le cuir), leur effet sur la matière et les besoins en restauration nécessaires ensuite peut aussi permettre de limiter ces traitements et réduire ainsi l'impact environnemental de notre activité.

\section{En conclusion}

De nombreuses pistes de réflexion et d'amélioration des pratiques existent aujourd'hui, explorées par tous les acteurs travaillant pour les institutions patrimoniales. La conservation du patrimoine doit plus que jamais prendre en compte les objectifs du développement durable et des compromis doivent absolument être trouvés. Des solutions doivent être apportées pour limiter l'impact d'activités comme le transport international d'œuvres d'art par exemple. Pour cela, la recherche en conservation est essentielle, mais on peut aussi s'inspirer d'autres milieux professionnels, comme le monde du spectacle notamment qui met en place depuis plusieurs années des actions intéressantes ${ }^{(9)}$. La rationalisation des pratiques, lévaluation raisonnée des risques, l'acceptation de conditions de conservation adaptées à la situation mais pas forcément « idéales sur le papier », l'emploi au plus juste des ressources sont autant de principes à mettre en place si l'on souhaite réunir les objectifs de la conservation et ceux du développement durable.

\section{Notes}

1) Rapport Brundtland, 1987.

(2) Code de Déontologie de l'ICOM, 2006

(3) Cibles d'éco-construction, éco-gestion, confort et santé

(4) Dorfman, É. Penser vert, Les Nouvelles de l'ICOM, n¹, 2011

(5) Aubert, S. Le développement durable dans la construction d'un Centre de Conservation et d'Étude, la Lettre de l'OCIM, n¹33, janvierfévrier 2011, pp. 18-23.

(6) L'empreinte écologique est un outil permettant de mesurer l'impact des activités humaines sur l'environnement.

(7) Michalski, S. Le climat dans les musées et le climat mondial, comment adopter des mesures appropriées aux deux, in Réflexions sur la conservation, Institut Canadien de Conservation, 2011.

(8) www.getty.edu/conservation/science/climate/climate_component3.html (9) Dossier «Spectacle et développement durable », La Scène, n 55 , hiver 2009-2010. 